\section{Nucleic acid synthesis}

\section{Pinyin tRNA}

\section{from Beverly Griffin and Tomas Lindahl}

A LARGE-SCALE research project conducted in the People's Republic of China has recently been brought to a successful conclusion. In the May 1983 issue of Scientia Sinica, a series of four papers (written in English) describe the total synthesis of a biologically active tRNA ${ }^{1,2}$. This is the first time such a molecule, indistinguishable from a natural isolate, has been synthesized.

The project was initiated as long ago as 1968 , but progress seems to have been relatively slow in the difficult research climate existing during the Cultural Revolution. Much of the work has been carried out at the Shanghai Institute of Biochemistry of the Academia Sinica by a research group directed by Wang Debao (who published as T.P. Wang before the current pinyin* transliteration system was generally adopted), but important contributions have also been made by other groups in Shanghai and Beijing. In fact, it is estimated by the Chinese workers that between 100 and 200 scientists have been involved in this project at various times, but this staggeringly large figure includes units such as the 'Shanghai No.2 Reagent Factory', which produces fine chemicals and precursors often readily available commercially to Western scientists.

While modified and improved chemical methods for DNA synthesis have made the production of oligodeoxyribonucleotides of defined sequence a widely used technique of molecular biology within the last few years, synthesis of related oligoribonucleotides is considerably more complicated because of the presence of reactive 2 '-OH groups on the sugar residues. Even today, it would seem forbiddingly difficult to try to synthesize a RNA molecule the size of a tRNA exclusively by chemical procedures. Therefore, the strategy finally adopted by the Chinese workers involved the chemical synthesis of several small oligoribonucleotides, which were then enzymatically joined together in a predetermined fashion. An analogous approach was pioneered for DNA by Khorana's group $^{3}$, in the first synthesis of an active gene.

The RNA molecule chosen for synthesis by the Chinese scientists was the alaninetRNA of baker's yeast, one of the very few complete RNA sequences known in 1968. This molecule contains 76 base residues, of which as many as 9 are modified versions of the four major nucleotide subunits (see the figure). Before synthesis, therefore, the necessary modifications had to be made to the monomer nucleotides by specific chemical reactions such as catalytic

*Pinyin is the standard system for transcribing Chinese characters into Roman ones. hydrogenation, methylation and deamination. One of the minor residues, pseudouridine, which is not readily obtainable by chemical means, was isolated from human urine and subsequently phosphorylated. The detailed procedures for the incorporation of such unusual, and in some cases labile, nucleotide derivatives into oligonucleotides still await publication.

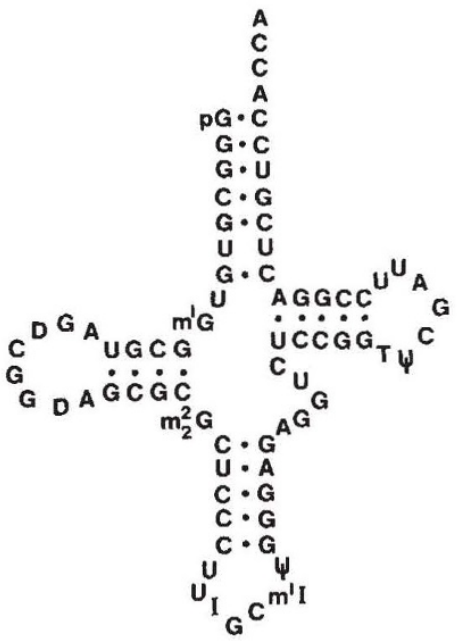

Primary structure of alanine-tRNA from baker's yeast. The nucleotide sequence is that proposed by Holley et al. ${ }^{8}$ with two subsequent minor revisions 9 . The modified nucleotide residues are $\mathrm{D}$, dihydrouridylic acid; $\psi$, pseudouridylic acid; I, inosinic acid; T, ribothymidylic acid; $m^{1} I, 1$-methylinosinic acid; $m^{1} G$, 1-methylguanylic acid; $\quad \mathrm{m}^{2}{ }_{2} \mathrm{G}$, 2-dimethylguanylic acid.

The discovery of the phage T4-induced RNA ligase ${ }^{4}$ and the demonstration that this enzyme effectively joins small singlestranded oligonucleotides together with no sequence-specificity or template requirements ${ }^{5}$ have been crucial for the synthesis of the tRNA in vitro. The other key enzyme used in the work was polynucleotide kinase, which allowed for the phosphorylation of $5^{\prime}$ ends with a ${ }^{32} \mathrm{P}$ radioactive marker. The latter enzyme normally also has an associated $3^{\prime}$-phosphatase activity, but the Chinese team used an additional mutant form of the enzyme that has only the kinase action. By ingenious use of these activities, it proved possible for the Chinese scientists to use $3^{\prime}$-phosphate residues as blocking groups during the ordered joining of oligonucleotides, subsequently removing the blocking moieties by enzymatic means.

Two of the four papers in Scientia Sinica describe the separate syntheses of the $5^{\prime}$ and $3^{\prime}$ halves of the tRNA Ala molecule ${ }^{1}$. In a preliminary study, it was shown by the Beijing group that half-molecules of tRNA Ala . obtained by limited ribonuclease
T1 cleavage of the molecule purified from yeast, can be efficiently rejoined with RNA ligase ${ }^{6}$. As detailed in the other two current papers ${ }^{1,2}$, the corresponding synthetic half-molecules were joined with RNA ligase, and the completed tRNA was shown to possess full amino-acid-acceptor activity, as well as transfer activity in vitro in a reticulocyte-derived protein-synthesis system. The synthetic molecules were also shown to migrate in the expected position on polyacrylamide gel electrophoresis in the presence of $7 \mathrm{M}$ urea. It is perhaps typical of the current preference for gel electrophoresis over centrifugal analysis that no attempt was made to show that the synthetic molecule had the $4 \mathrm{~S}$ sedimentation coefficient characteristic of the native compact tRNA conformation.

The principal difference between this impressive synthesis and earlier efforts to fabricate tRNA in vitro is the inclusion of all the correct modified residues in the synthetic molecule. Ohtsuka et al. ${ }^{7}$ have previously reported the synthesis of an RNA molecule with a sequence related to that of Escherichia coli formylmethioninetRNA, using an approach similar to that adopted in Shanghai. Although the work is very elegant, their final product was entirely composed of unmodified residues and showed little amino-acid-acceptor activity. Similarly, when the synthetic gene for an E. coli tyrosine-tRNA was transcribed in vitro, the transcript was inactive in an amino-acid-acceptor assay, although the gene could be shown to be active in vivo ${ }^{3}$. Taken together, these data dramatically demonstrate the crucial role of the modified nucleotides in conferring biological activity on tRNA molecules.

With the present achievements in synthesis, and the availability of the appropriate building blocks, the road now seems open for an unequivocal assignment of the relative roles of various base modifications for enzyme recognition or as key structural features in tRNA, since one after another of the altered residues can now be replaced by its unmodified counterpart in a synthetic molecule which can then be assayed for biological activity. This promising line of research is now being pursued by the Shanghai group.

Beverly Griffin and Tomas Lindahl are with the Imperial Cancer Research Fund, Lincoln's Inn Fields, London WC2A $3 P X$.

. Wang Debac et al. Scientia sin. B26, 464, 482, 495 (1983).

Shen Qingxiang el al. Scientia sin. B26, 504 (1983).

. Sekiya, T. et al, J. biol. Chem. 254, 5787, 5802 (1979).

. Silber, R., Malathi, V.G. \& Hurwiz, J. Proc. nutn. Acad. Sci. U.S. A. 69. 3009 (1972).

5. Walker, G.C. et al. Proc. natn. Acad. Sci. U.S. A. 72, 122 (1975).

6. Wang Gui-Hai et al, Biochim. biophys. Acta 652,82 (1981).

7. Ohtsuka, E et al. Proc. natn. Acad, Sci, U.S. A. 78, 5493 (1981).

8. Hollcy, R.W. et al. Science 174, 1462 (1965).

9. Penswick, I.R., Martin, R. \& Dirheimer, G. FEBS Lelt. 50, 28 (1975). 